\title{
Two cases of serotypeable and non- serotypeable variants of Streptococcus pneumoniae detected simultaneously during invasive disease
}

Kedibone M. Ndlangisa ${ }^{1,2^{*}}$, Mignon du Plessis ${ }^{1,2}$, Mushal Allam ${ }^{1}$, Nicole Wolter ${ }^{1,2}$, Thabo Mohale ${ }^{1,2}$, Linda de Gouveia', Monica Birkhead ${ }^{3}$, Keith P. Klugman ${ }^{4}$ and Anne von Gottberg ${ }^{1,2}$

\begin{abstract}
Background: More than 94 serotypes of Streptococcus pneumoniae have been described to date, however the majority of disease is caused by approximately 20 serotypes. Some pneumococci do not react with commercially available antisera used for serotyping and are thus regarded as non-serotypeable (NT). These pneumococci are commonly isolated during carriage studies and very rarely cause invasive disease. Colonization may occur with more than one serotype however disease with more than one serotype is rarely detected. Thus there are limited data describing cases of pneumococcal disease caused by more than one isolate.

Results: In two cases of invasive pneumococcal disease in South Africa, a non-serotypeable and a serotypeable isolate were co-detected during routine serotyping. A serotype 1 and $18 \mathrm{C}$ isolate were each co-detected with a non-serotypeable isolate in 2009 (case A) and 2010 (case B), from cerebrospinal fluid and blood, respectively. Both patients were 10-14 years old. For case A, the serotypeable isolate could not be obtained due to low representation in the mixed culture. Using electron microscopy we confirmed lack of capsule for the non-serotypeable isolates. Comparison of the case $A$ non-serotypeable isolate with a serotype 1 genome revealed only the presence of the rhamnose biosynthesis genes $(r m / A, B, C$ and $D)$ in the capsular locus, all other capsular genes were absent. Nonetheless it had a multilocus sequence type (ST) associated with serotype 1 (ST217 and ribosomal ST3462) and its core genome clustered with other ST217 isolates. The case B non-serotypeable isolate had all serotype 18C capsular genes except for variation in the wchA and wze genes, compared to the 18C isolate. Both case B isolates were ST9817 and their core genomes were identical.
\end{abstract}

Conclusions: The ability of pneumococci to alter capsule production is a potential vaccine escape mechanism and therefore non-serotypeable pneumococci should be monitored as such organisms may increase under vaccine pressure.

Keywords: wchA, cpsE, Non-serotypeable, Serotype 1, Serotype 18C, Pneumococcus, Mixed culture, South Africa

\footnotetext{
* Correspondence: kedibonen@nicd.ac.za

${ }^{1}$ Centre for Respiratory Diseases and Meningitis (CRDM), National Institute for

Communicable Diseases (NICD), a division of the National Health Laboratory

Service, Johannesburg, South Africa

${ }^{2}$ School of Pathology, University of the Witwatersrand, Johannesburg, South

Africa

Full list of author information is available at the end of the article
} 


\section{Background}

Streptococcus pneumoniae (pneumococcus) is a commensal of the human nasopharynx. However, occasionally it evades the immune system and is an important cause of invasive disease such as meningitis and bacteraemia, and non-invasive disease such as pneumonia and acute otitis media. The polysaccharide capsule is known to be a major virulence factor of the pneumococcus. It is immunogenic and can be detected by specific antisera, and hence forms the basis for serotyping and current vaccines against pneumococcal disease. To date, more than 94 serotypes have been described but the majority of disease is caused by approximately 20 serotypes [1]. The most prevalent disease-causing serotypes have been incorporated into currently used polysaccharide conjugate vaccines (PCV-7, -10 and -13). In South Africa, PCV-7 was introduced into the Expanded Programme for Immunisation in 2009 and replaced by PCV-13 in 2011 in a $2+1$ schedule at 6,14 and 40 weeks of age [2].

With the exception of serotypes 3 and 37, genes responsible for production of the polysaccharide capsule are located in the capsular polysaccharide (cps) region in a single locus flanked by the $\operatorname{dex} B$ and aliA genes [3]. The first four cps genes (cpsA, cpsB, cpsC and cpsD also known as $w z g, w z h$, wzd and wze, respectively) are conserved among all serotypes and are involved in the regulation and processing of the capsule [3]. Some pneumococci do not react with commercially available antisera used for serotyping and are thus regarded as non-serotypeable (NT). These pneumococci are commonly isolated during carriage studies and very rarely cause invasive disease [4]. Non-serotypability is due to partial or complete loss of the cps gene cluster [5], replacement of cps genes with other genes [5-7], sequence duplication [8] or single point mutations, commonly within the $w c h A$ (also known as cpsE) gene $[9,10]$. The polysaccharide capsule plays an important role in the pathogenesis of the pneumococcus as it provides protection against complement-mediated phagocytosis. Reduced levels of capsule expression are required for effective attachment to epithelial cells and colonization [11].

While reviewing national surveillance data to identify episodes of mixed invasive pneumococcal disease (IPD) caused by two or more serotypes, we identified two cases (subsequently referred to as case A and case B) whereby one of the co-detected isolates was non-serotypeable (NT). In each of the two cases, the serotypeable and the NT variant was identified from a culture recovered from the same specimen (cerebrospinal fluid for case A and blood for case B). Given the rarity of NT isolates in causing IPD we hypothesized that these NT isolates were variants of their co-detected serotypeable isolates. We therefore compared the genomes of these co- detected NT and serotypeable isolates to determine their relationships.

\section{Results}

Thirty-seven thousand eight hundred twenty-five cases of IPD were reported from 2005 to 2013, of which $70 \%$ $(26,475 / 37,825)$ had viable isolates. Serotype results were available for nearly all cases with viable isolates $(26,469 /$ 26,475). A mixed culture with more than one serotype detected (including non-serotypables) was identified for $41(0.2 \%)$ cases. In two cases, one of the co-detected isolates was NT. These cases were reported in 2009 (case A) and 2010 (case B), respectively and the age range for both was 10-14 year-old (Table 1). These two mixed culture cases were identified during routine serotyping of cultures recovered from cerebrospinal fluid (case A) and blood (case B). Case A was diagnosed with meningitis and clinical information was not available for case $\mathrm{B}$ to confirm diagnosis.

For case A, serotype 1 and NT S. pneumoniae were identified from the same culture, with the NT isolate representing approximately $98 \%$ of the culture (using the Quellung reaction). The case B isolates were identified as NT and 18C. In each of the two cases, the same results were obtained by two different laboratory staff performing the Quellung reaction on fresh overnight cultures grown from the original transport medium. After several attempts to separate the two variants, only the NT isolate could be obtained for case A, however, real-time PCR confirmed the presence of the serotype 1 wzy gene in the mixed culture. For case $\mathrm{B}$, pure cultures were obtained for both the $18 \mathrm{C}$ and the NT isolate. The three isolates were susceptible to all tested antimicrobial agents.

TEM confirmed the absence of capsular material for the case A non-serotypeable isolate (Fig. 1). For

Table 1 Characteristics of two patients (case A and B) with invasive Streptococcus pneumoniae serotypeable and nonserotypeable co-disease, South Africa

\begin{tabular}{lll}
\hline & Case A & Case B \\
\hline Age range in years & $10-14$ & $10-14$ \\
Gender & male & male \\
HIV status & unkown & unknown \\
Specimen & cerebrospinal fluid & blood \\
Year identified & 2009 & 2010 \\
Diagnosis & meningitis & unknown \\
Co-detected serotypes & non-serotypeable & non-serotypeable \\
& and serotype 1 & and serotype 18C \\
Isolated serotypes & anon-serotypeable & non-serotypeable \\
& & and 18C
\end{tabular}

aPure culture for serotype 1 could not be obtained due to its low representation in the mixed culture (see Results for more information) 


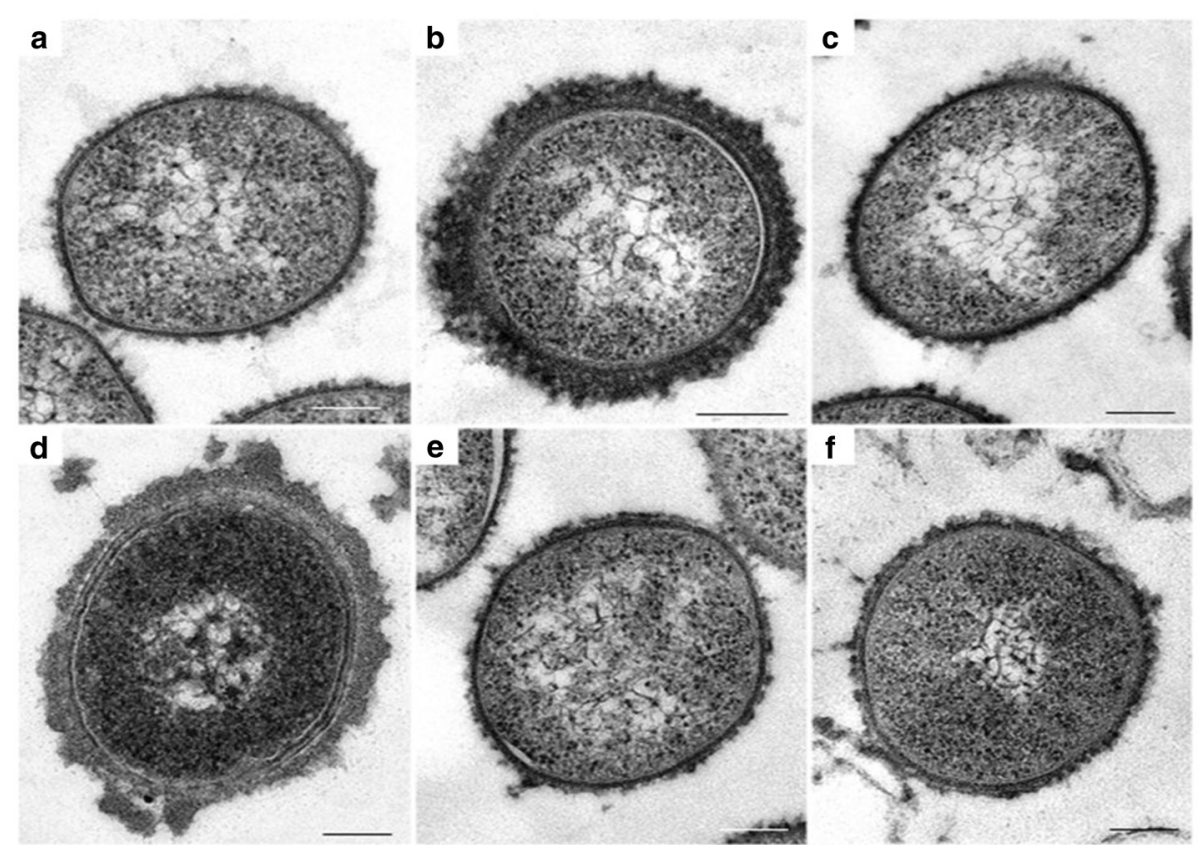

Fig. 1 Visualization of pneumococcal isolates using transmission electron microscopy (TEM). Capsular materials of non-serotypable pneumococcal isolates causing mixed infections in two patients in South Africa were compared to capsular materials of serotypeable isolates. The two cases were reported in 2009 (case A) and 2010 (case B). For case A non-serotypeable and a serotype 1 isolate were identified and for case B a non-serotypeable and $18 \mathrm{C}$ isolates were identified. TEM of the case A non-serotypeable isolate is shown in $\mathbf{a}$, TEM of serotype 1 clinical isolate used as a control in $\mathbf{b}$, TEMs of two non-serotypeable clinical isolates used as a controls in $\mathbf{c}$ and $\mathbf{f}$, TEM of case B serotype 18C isolate in $\mathbf{d}$, and case B non-serotypeable isolate in e. Scale bar $=175 \mathrm{~nm}$

case B, 50 to $120 \mathrm{~nm}$ thick capsular material was observed around cells of the serotype $18 \mathrm{C}$ isolate, while there was no capsular material identified for the NT isolate (Fig. 1).

The genome coverage for the case A NT isolate was $320 \times$ and assemblies contained 33 contiguous sequences with a total length of 2,023,465 bp. For the case B NT isolate, the coverage was $355 \times$ and assemblies contained 91 contiguous sequences with a total length of 2,110,682 bp. The case B serotype 18C isolate genome coverage was $310 \times$ and assemblies contained 78 contiguous sequences with total a length of 2,115,055 bp.

Sequence analysis of the capsular locus of the case A NT isolate revealed the absence of all but four capsular genes [rhamnose biosynthesis genes $(r m l A, B, C$ and $D)$ ] (Fig. 2). The isolate was identified as ST217 and rST3462. Because we did not obtain a pure culture for the case A serotype 1 isolate, we compared the case A NT genome sequence to genomes $(n=54)$ of ST217 serotype 1 isolates causing invasive disease in South Africa available on S. pneumoniae PubMLST database [12] to further confirm that this NT isolate was indeed a serotype 1 variant. Except for the absence of genes in the capsular region (Additional file 1: Figure S1); the NT core genome was $100 \%$ similar (in $100 \%$ of the bootstrap replications) to sequences of a serotype 1 isolate with the same rMLST profile (rST3462) (Additional file 2: Figure S2). It also clustered with ST217 clinical isolates collected from 1989 to 2013.

For case B, the NT and $18 \mathrm{C}$ isolates shared the same new ST (ST9817) and rMLST profile. The NT isolate had all serotype 18C capsule-specific genes [3], however variations were identified in the capsular locus compared to the co-detected $18 \mathrm{C}$ isolate and three other genotypically related invasive $18 \mathrm{C}$ isolates from South Africa. A nucleotide insertion (472_473 insG) that resulted in a translational frameshift, introducing a stop codon in the $w c h A$ gene, was identified (Additional file 3: Figure S3). A substitution $(149 \mathrm{C} \rightarrow \mathrm{A})$ in the wze gene resulted in an amino acid change (T50K) (Additional file 4: Figure S4). Genome sequence comparison of the case B isolates revealed high similarity between the two genomes (Additional file 5: Figure S5) with $100 \%$ identity between their core genomes (Additional file 6: Figure S6).

\section{Discussion}

The polysaccharide capsule is an important component of the pneumococcus that aids in evading the immune system. Hence unencapsulated (non-serotypeable) 


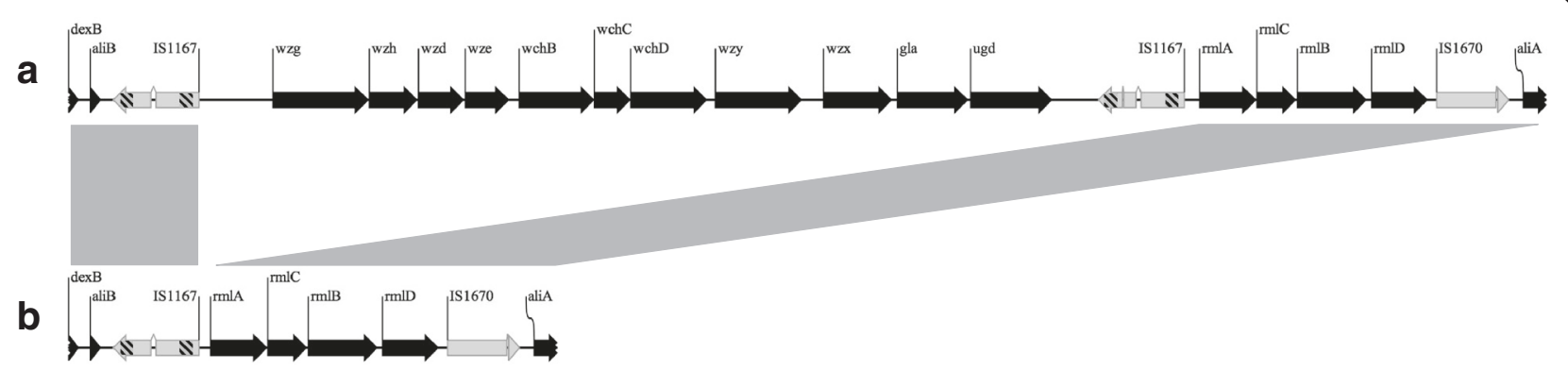

Fig. 2 Schematic diagram representing capsular polysaccharide loci of a pneumococcal non-serotypeable and serotype 1 isolate. The schematic diagram represents comparison between the capsular polysaccharide (cps) locus of a non-serotypeable isolate (b) co-detected with a serotype 1 isolate during an invasive disease episode in South Africa, 2009, and the cps locus of an invasive serotype 1 clinical isolate from South Africa (a). Transposases are indicated by grey arrows and flanking repeated sequences by lines within the arrow delimitations

pneumococci rarely cause invasive disease. Nevertheless, we describe two cases of invasive pneumococcal disease caused simultaneously by what appears to be serotypeable and non-serotypeable variants of the same strain. Our hypothesis, that the NT isolates were variants of their encapsulated counterparts that either lost their capsules or had reduced levels of capsular expression during the infection, is supported by the lack of capsule for both NT isolates from the two cases. Both NT isolates were sequence types of major clones circulating among encapsulated isolates in South Africa [13]. One NT isolate was ST217, the dominant serotype 1 clone in South Africa [13]. The second isolate was a new sequence type (ST9817) that belonged to the ST1016 clonal complex, a predominant clonal complex among serotype 18C isolates in South Africa [13].

Since the NT isolates from both cases were sequence types commonly associated with serotype 1 and $18 \mathrm{C}$, we aligned their cps sequences to that of serotype 1 and $18 \mathrm{C}$, respectively. The non-serotypability of the ST217 isolate appears to be caused by the deletion of genes within the cps region. This mechanism is common among NT isolates with a serotype 1 genetic background. Scott et al. [14], analysed 28 NT isolates from IPD patients and identified eight isolates with partial deletion of the capsular locus. Four of the eight isolates were a sequence type associated with serotype 1 (ST227) and, similar to our ST217 isolate, only had the rhamnose biosynthesis genes ( $r m l A, B, C$ and $D)$ in their capsular region. Similarly, four IPD NT isolates analysed by Salter et al. [15] that were ST227 only had $\operatorname{rml} A, B, C$ and $D$ present in their capsular locus. Five additional ST217 NT isolates that were identified among our collection of IPD surveillance isolates and were analysed as part of another study also had only the four rhamnose biosynthesis genes present in the capsular locus (unpublished data). The deletion of the cps genes in our ST217 nonserotypeable isolate could be transposon mediated as the deleted serotype 1 genes are flanked by transposase genes (IS1167). We were unable to isolate the encapsulated variant (serotype 1) from this case due to its low representation (approximately $2 \%$ ) in the mixed culture. Nevertheless, detection of the serotype 1 wzy gene (absent in the genome sequence of the NT isolate) in the mixed culture by real-time PCR supports the presence of serotype 1 in that culture. Our genotypic results also confirm that the NT isolate is a serotype 1 variant as both the sequence type and ribosomal MLST profile are exclusively associated with serotype 1 (ST217 and rST3462). In addition, phylogenetic analysis showed that its core genome clustered with those of other invasive ST217 serotype 1 isolates circulating in South Africa during the same period.

In the second case, the NT isolate had an intact cps locus with all serotype $18 \mathrm{C}$ capsular genes with the exception of two capsular genes that were variable, compared to the serotype $18 \mathrm{C}$ isolate from the same patient. Variation in the wze gene resulted in an amino acid change within the protein. Together with $w z d$, wze encodes proteins responsible for the export of the mature polysaccharide to the cell surface [3]. The wze gene encodes an auto-phosphorylating protein-tyrosine kinase which is inhibited by aerobic microenvironments, and is thought to be one of the factors causing decreased capsular expression in host environments with higher oxygen levels such as the nasopharynx $[11,16]$. Previous studies have demonstrated that mutations in the wze gene can inhibit capsule production [11, 16, 17]. An insertion that introduced a premature stop codon in the $w c h A$ gene was identified in our NT isolate. We suspect that this insertion may be responsible for the loss of capsule expression in our isolate as $w c h A$ encodes the initial transferase that catalyzes the transfer of glucose-phosphate to the lipid carrier during capsular polysaccharide biosynthesis [18]. Melchior et al. [19] described two mutations in the wchA gene (a 
nucleotide deletion at position 910 and a substitution at position 1081) in two invasive non-serotypeable isolates with serotype $7 \mathrm{~F}$ capsular genes both of which introduced premature stop codons in the wchA gene. Although the positions of these mutations differed from that of our isolate, both resulted in a premature stop codon. In another study, Schaffner et al. [9] recovered a NT isolate, with serotype 18C capsular locus, from the nasopharynx of a child with otitis media. The variations identified in our NT isolate with serotype $18 \mathrm{C}$ capsular genes differ from the wchA single base substitution (C1135G) described in the Schaffner study.

Repeated sub-culturing in the laboratory may lead to a change in phenotype [20] or the cultures may have become mixed as a result of laboratory error. It is unlikely that this occurred in this instance given the rarity of these cases in our laboratory and the correlating genotypic data between the case $\mathrm{B}$ variants. It is favourable for the pneumococcus to have reduced levels of capsule for effective attachment to epithelial cells in the nasopharynx however the capsule is required for survival of the pneumococcus during invasive disease as it provides protection against complement-mediated phagocytosis [11]. We therefore suspect that both variants may have been present in the nasopharynx and invaded simultaneously. Unfortunately we don't have corresponding nasopharyngeal isolates from these patients to confirm this hypothesis. Another possibility is that the serotypeable variant could have invaded and mutated in the host to lose the capsule. Survival of the NT isolates in the blood and meninges without protection of the capsule is highly irregular but may be related to host factors such as suppressed immune status due to underlying diseases such as HIV infection [21]. Unfortunately we did not have all the relevant patient information to assess if this was true.

\section{Conclusions}

We identified two different vaccine-serotype isolates that appear to have lost their capsules during two separate episodes of invasive disease. Non-serotypeable pneumococci are not targeted by current pneumococcal vaccines and thus present a potential mechanism whereby the organism could escape vaccine pressure. In addition, unencapsulated isolates have higher recombination efficiency than encapsulated pneumococci and therefore provide a reservoir of antibiotic resistance genes for genetic exchange [22]. Antibiotics coupled with routine PCV use may drive the selection of non-serotypeable invasive variants and thus prevalence and genotypes of such pneumococci should be monitored to identify capsular switching or other genetic exchange that may occur under vaccine pressure.

\section{Methods}

\section{Bacterial strains}

Isolates were obtained through Group for Enteric, Respiratory and Meningeal Disease Surveillance (GERMS-SA), a national, laboratory-based surveillance system for IPD in South Africa [23]. Identification of S. pneumoniae was based on standardized methodologies using optochin as well as bile solubility on any optochin nonsusceptible strains [24]. Once identified, a sweep was inoculated onto Dorset transport medium [Diagnostic Media Products (DMP), Johannesburg, South Africa] and sent to the NICD [25]. Isolates were sub-cultured on arrival at our reference laboratory on $5 \%$ horse blood agar plates (DMP) in the presence of an optochin disc (Mast Group Ltd., United Kingdom). Cultures were stored in $10 \%$ skim milk (DMP) at $-70{ }^{\circ} \mathrm{C}$.

\section{Serotyping and antimicrobial susceptibility testing}

Serotypes were determined by the Quellung method using serotype-specific antisera (Statens Serum Institut, Copenhagen, Denmark) [26]. A sweep of culture was initially serotyped and if a mixed culture was suspected, serotyping was repeated on single colonies. Antimicrobial susceptibility testing was performed by the broth microdilution method using commercially prepared Sensititre-SASP2 panels (Trek Diagnostics Inc., Cleveland, $\mathrm{OH}$ ). Results were interpreted according to Clinical and Laboratory Standards Institute guidelines and breakpoints [27]. The following antimicrobial agents were tested: penicillin G, ceftriaxone, amoxicillin, erythromycin, clindamycin, chloramphenicol, tetracycline, rifampicin, cotrimoxazole, ofloxacin, linezolid and vancomycin. Isolates were considered to be non-susceptible to penicillin at minimum inhibitory concentrations (MICs) $\geq 0.12 \mathrm{mg} /$ $\mathrm{L}$ using the oral penicillin meningitis breakpoints. For other antimicrobials, isolates were defined as nonsusceptible if they were intermediately or fully resistant to the agent tested.

\section{Identification of cases with mixed cultures of a non-serotypeable and a serotypeable isolate}

Cases reported for IPD surveillance from 2005 to 2013 were reviewed. A mixed culture was defined as the simultaneous identification of at least two serotypes (including non-serotypeable isolates) either from the same normally-sterile site specimen of an IPD patient or from two or more normally-sterile site specimens obtained from an IPD patient within 21 days of each other. In this study, we characterized isolates from cases where at least one of the co-identified isolates was non-serotypeable. Such cases were detected during routine serotyping by Quellung if an isolate reacted partially with a specific antiserum pool. In such cases, the stored isolate and 
Dorset transport medium were sub-cultured for single colonies on $5 \%$ horse blood agar plates (DMP). Following $24 \mathrm{~h}$ incubation at $37{ }^{\circ} \mathrm{C}$ in $5 \% \mathrm{CO}_{2}$, the plates were examined for differences in colony morphology and the Quellung reaction was repeated on different colony types. The colonies were sub-cultured, re-identified and serotyped to determine if they were true mixtures. Real-time PCR, targeting the lytA gene, was used to confirm non-serotypeable isolates as $S$. pneumoniae [28]. Non-serotypeable isolates were defined as pneumococcal isolates confirmed by lyt $A$ real-time PCR and for which a serotype could not be assigned by the Quellung method. For the serotypeable isolate and mixed cultures where isolates of different serotypes could not be separated, serotype was confirmed using real-time PCR [29].

\section{Transmission electron microscopy (TEM)}

TEM was conducted to visualize the presence of capsular material. Isolates from overnight cultures were fixed in situ on agar plates, and processed according to the protocol developed by Hammerschmidt et al. with ruthenium red and L-lysine acetate fixation [30]. In addition, three clinical isolates with confirmed serotyping results (one serotype 1 and two NT) were selected from the GERMS-SA collection and were included as controls for TEM analysis.

\section{Genome sequencing}

Overnight fresh cultures were inoculated into brain heart infusion broth (DMP) and incubated overnight at $37{ }^{\circ} \mathrm{C}$ in $5 \% \mathrm{CO}_{2}$. Cells were pre-lysed at $37^{\circ} \mathrm{C}$ for $1 \mathrm{~h}$ in $10 \mathrm{mg} / \mathrm{ml}$ of lysozyme (Sigma-Aldrich, St. Louis, MO) and DNA was extracted using the QIAamp DNA mini kit (Qiagen, Venlo, Netherlands). DNA extracts were quantified using the Qubit instrument and dsDNA BR Assay kit (Life Technologies, Carlsbad, CA, USA). Multiplexed paired-end libraries were prepared using the Nextera XT DNA sample preparation kit (Illumina, San Diego, CA, USA). Genome sequencing was carried out on an Illumina MiSeq platform. Each isolate was extracted and sequenced three times, each time from a fresh culture.

\section{Genome analysis}

The paired-end reads were checked for quality, trimmed and de novo assembled using the Qiagen CLC Genomics Workbench version 8 (Qiagen, Venlo, Netherlands). The resultant contiguous sequences were then ordered using Mauve [31] and S. pneumoniae ATCC 700669 as a reference [GenBank:FM211187] and annotated using Prokka version 1.11 [32]. In addition the sequenced reads were mapped against references for capsular regions of co-detected serotypes [GenBank: CR931632 and CR931673].

Variations within the capsular polysaccharide biosynthesis genes were detected using the fixed ploidy variant detection tool within the CLC Genomics Workbench. This model detects the variants whose representation in the reads is in accordance with the assumed ploidy, discards variants whose representation in the reads is likely due to sequencing errors or mapping artefacts and then reports on positions where there may be single nucleotide variation (SNV) including insertions, deletions and substitutions. Identified variations were further investigated using CLC Genomic workbench to identify amino acid changes.

Sequences of the 7 multilocus sequence typing (MLST) genes were extracted from the assembled genomes and allele numbers and sequence types (ST) assigned using the Bio-MLST-Check module (http:// search.cpan.org/dist/Bio-MLST-Check/lib/Bio/MLST/ Check.pm). Furthermore, contiguous sequences were uploaded to the S. pneumoniae PubMLST isolates database [12], which runs the Bacterial Isolate Genome Sequence database (BIGSdb) platform to facilitate the ribosomal MLST (rMLST) analysis [33]. Phylogenetic similarity analysis, based on the core genomes, was performed using the Rapid large-scale prokaryote pan genome analysis (Roary) [34] and the maximum likelihood trees were generated using RaxML version 8 [35]. The genomes of case A and case B isolates were compared to serotype $1(n=54)$ and $18 \mathrm{C}(n=58)$ genomes randomly selected from sequences available on $S$. pneumoniae PubMLST database [12]. The selected sequences were of serotype 1 and $18 \mathrm{C}$ isolates that caused IPD in South Africa from 1989 to 2013 and 2005 to 2013, respectively. For the analysis, core genomes consisted of genes that were present as single copies in at least $90 \%$ of isolates being analysed and, in addition, had the same sequence length in every isolate. The BLAST Ring Image Generator (BRIG) was used to visualise similarities between genomes of mixed culture isolates [36].

\section{Additional files}

Additional file 1: Figure S1. BLAST Ring Image of the complete genome sequence of a non-serotypeable and serotype 1 isolate. This figure shows comparison between genome sequence of a non-serotypeable (NT) isolate co-detected with a serotype 1 isolate during a single episode of invasive disease in South Africa in 2009 and genome sequences of a serotype 1 isolate of the same sequence type (ST) 217 and ribosomal ST3462 as the NT isolate. (PDF $191 \mathrm{~kb}$ )

Additional file 2: Figure S2. Core genome phylogenetic tree of Case A non-serotypeable isolate and serotype 1 isolates from South Africa. The tree represents phylogenetic comparison of the core genome of a nonserotypeable (NT) co-detected with a serotype 1 isolate in 2009 in South Africa and genomes of serotype 1 isolates $(n=54)$ that caused invasive disease in South Africa from 1989 to 2013. This maximum likelihood tree 
was built using core gene sequences which were concatenated and aligned, using MUSCLE (EMBL-EBI). The numbers at the nodes indicate RAxML bootstrap values. Branch lengths are proportional to the number of substitutions per site (see the scale bars). The colour coding is according to sequence type (ST). (PDF $173 \mathrm{~kb}$ )

Additional file 3: Figure S3. Comparison of $w C h A$ gene sequences of $18 \mathrm{C}$ and non-serotypeable isolates from a South African patient. Amino acid sequences of wchA genes of two isolates [serotype 18C and nonserotypeable (NT)] recovered simultaneously from a patient with invasive pneumococcal disease in South Africa in 2010 were aligned to identify differences between the two sequences. The region where the NT amino acid sequence differs from the $18 \mathrm{C}$ sequence is enclosed in a box. Position of a stop codon within the NT amino acid sequence is indicated by an asterisk (*). (PDF $150 \mathrm{~kb}$ )

Additional file 4: Figure S4. Comparison of wze gene sequences of $18 \mathrm{C}$ and non-serotypeable isolates from a South African patient. This figure shows amino acid sequence alignment of wze genes of two isolates [serotype 18C and non-serotypeable (NT)] recovered from a patient with invasive pneumococcal disease in South Africa is shown. The box indicates a variable amino acid due to a single nucleotide variation between the two isolates. (PDF $177 \mathrm{~kb}$ )

Additional file 5: Figure S5. BLAST Ring Image (BRIG) of the complete genome sequence of a non-serotypeable and serotype $18 \mathrm{C}$ isolate. This figure visualise similarities between genomes of mixed culture isolates. The two isolates [serotype $18 \mathrm{C}$ and non-serotypeable (NT)] were codetected during a single episode of invasive disease in South Africa in 2010. (PDF $197 \mathrm{~kb}$ )

Additional file 6: Figure S6. Core genome phylogeny of a nonserotypeable isolate and serotype $18 \mathrm{C}$ isolates from South Africa. The tree represents phylogenetic comparison of the core genome of two isolates that were co-detected during a single episode of invasive disease in South Africa in 2010 [serotype 18C and non-serotypeable (NT)] and genomes of serotype $18 \mathrm{C}$ isolates $(n=58)$ that caused invasive disease in South Africa from 2005 to 2013. This maximum likelihood tree was built using core gene sequences which were concatenated and aligned, using MUSCLE (EMBL-EBI). The numbers at the nodes indicate RAXML bootstrap values. Branch lengths are proportional to the number of substitutions per site (see the scale bars). The colour coding is according to sequence type (ST). (PDF 164 kb)

\section{Abbreviations}

GERMS-SA, Group for Enteric, Respiratory and Meningeal Disease Surveillance; S. pneumoniae, Streptococcus pneumoniae; IPD, invasive pneumococcal disease; HIV, human immunodeficiency virus; $\mathrm{PCV}$, polysaccharide conjugate vaccine; $\mathrm{CO}_{2}$, carbon dioxide; DMP, Diagnostic media production; MIC, minimum inhibitory concentration; PCR, polymerase chain reaction; NT, non-serotypeable; cps, capsular polysaccharide; TEM, transmission electron microscopy; Roary, Rapid large-scale prokaryote pan genome analysis; MLST, multilocus sequence typing; IMLST, ribosomal multilocus sequence typing; ST, sequence type; BIGSdb, Bacterial Isolate Genome Sequence database; SNV, single nucleotide variation; BRIG, BLAST Ring Image Generator

\section{Acknowledgements}

We thank GERMS-SA (Group for Enteric, Respiratory and Meningeal Surveillance) for coordinating national surveillance of IPD in South Africa. We are grateful to CRDM laboratory staff for their technical input and all clinical and laboratory staff throughout South Africa for submitting case reports and isolates for the GERMS-SA national surveillance program, and all patients whose isolates were used in the study.

\section{Funding}

The surveillance study was funded by NICD/National Health Laboratory Service, South Africa; PEPFAR (President's Emergency Plan for AIDS Relief) through the Centers for Disease Control and Prevention (cooperative agreement No. U62/ CCU022901. Genome sequencing was funded by Pfizer South Africa [grant number:WS116752(1)].

\section{Availability of data and materials}

The assembled genomes of case A NT isolate, case B 18C isolate and case B NT isolate have been deposited in the NCBI database [GenBank:LHAG00000000, LGHY00000000 and LHAF00000000, respectively].

\section{Authors' contributions}

$\mathrm{KN}, \mathrm{MdP}, \mathrm{AvG}, \mathrm{NW}, \mathrm{TM}$ and KK contributed to the conception and design of the study. KN carried out DNA extractions and drafted the manuscript. LdG performed serotyping and antimicrobial susceptibility testing, MA performed genome analysis. MB conducted transmission electron microscopy. All authors read and approved the manuscript.

\section{Competing interests}

The authors declare that they have no competing interests.

\section{Consent for publication}

Consent to publish was obtained as part of ethics for the national surveillance study (protocol number: M081117).

\section{Ethics approval and consent to participate}

Ethical approval for this study was obtained from the Human Research Ethics Committee: (Medical), University of the Witwatersrand, Johannesburg, South Africa (protocol numbers: M111008 and M081117, respectively). Consent for patients' participation and collection of clinical data was obtained as part of ethics for the national surveillance study (protocol number: M081117).

\section{Author details}

${ }^{1}$ Centre for Respiratory Diseases and Meningitis (CRDM), National Institute for Communicable Diseases (NICD), a division of the National Health Laboratory Service, Johannesburg, South Africa. ${ }^{2}$ School of Pathology, University of the Witwatersrand, Johannesburg, South Africa. ${ }^{3}$ Centre for Emerging and Zoonotic Diseases, National Institute for Communicable Diseases (NICD), a division of the National Health Laboratory Service, Johannesburg, South Africa. ${ }^{4}$ Hubert Department of Global Health, Rollins School of Public Health, and Division of Infectious Diseases, School of Medicine, Emory University, Atlanta, GA, USA.

Received: 24 October 2015 Accepted: 15 June 2016

Published online: 24 June 2016

\section{References}

1. Johnson HL, Deloria-Knoll M, Levine OS, Stoszek SK, Freimanis HL, Reithinger $R$, Muenz LR, O'Brien KL. Systematic evaluation of serotypes causing invasive pneumococcal disease among children under five: the pneumococcal global serotype project. PLoS Med. 2010;7, e1000348.

2. Madhi SA, Cohen C, von Gottberg A. Introduction of pneumococcal conjugate vaccine into the public immunization program in South Africa: translating research into policy. Vaccine. 2012;30 Suppl 3:C21-7. doi:10.1016/j.vaccine.2012. 05.055:.C21-C27.

3. Bentley SD, Aanensen DM, Mavroidi A, Saunders D, Rabbinowitsch E, Collins M, Donohoe K, Harris D, Murphy L, Quail MA, Samuel G, Skovsted IC, Kaltoft MS, Barrell B, Reeves PR, Parkhill J, Spratt BG. Genetic analysis of the capsular biosynthetic locus from all 90 pneumococcal serotypes. PLoS Genet. 2006;2, e31.

4. Andrade AL, Franco CM, Lamaro-Cardoso J, Andre MC, Oliveira LL, Kipnis A, Rocha CG, Andrade JG, Alves SL, Park IH, Nahm MH, Almeida SG, Brandileone MC. Non-typeable Streptococcus pneumoniae carriage isolates genetically similar to invasive and carriage isolates expressing capsular type 14 in Brazilian infants. J Infect. 2010:61:314-22.

5. Park IH, Kim KH, Andrade AL, Briles DE, McDaniel LS, Nahm MH. Nontypeable pneumococci can be divided into multiple cps types, including one type expressing the novel gene pspK. MBio. 2012;3:e00035-12.

6. Hathaway LJ, Stutzmann MP, Battig P, Aebi S, Muhlemann K. A homologue of aliB is found in the capsule region of nonencapsulated Streptococcus pneumoniae. J Bacteriol. 2004;186:3721-9.

7. Hilty M, Wuthrich D, Salter SJ, Engel H, Campbell S, Sa-Leao R, de LH, Hermans P, Sadowy E, Turner P, Chewapreecha C, Diggle M, Pluschke G, McGee L, Koseoglu EO, Low DE, Smith-Vaughan H, Endimiani A, Kuffer M, Dupasquier M, Beaudoing E, Weber J, Bruggmann R, Hanage WP, Parkhill J, Hathaway LJ, Muhlemann K, Bentley SD. Global phylogenomic analysis of nonencapsulated Streptococcus pneumoniae reveals a deep-branching 
classic lineage that is distinct from multiple sporadic lineages. Genome Biol Evol. 2014;6:3281-94.

8. Waite RD, Penfold DW, Struthers JK, Dowson CG. Spontaneous sequence duplications within capsule genes cap8E and tts control phase variation in Streptococcus pneumoniae serotypes 8 and 37. Microbiology. 2003;149:497-504.

9. Schaffner TO, Hinds J, Gould KA, Wuthrich D, Bruggmann R, Kuffer M, Muhlemann K, Hilty M, Hathaway LJ. A point mutation in cpsE renders Streptococcus pneumoniae nonencapsulated and enhances its growth, adherence and competence. BMC Microbiol. 2014;14:210. doi:10.1186/ s12866-014-0210-x.:210-0210.

10. Park IH, Geno KA, Sherwood LK, Nahm MH, Beall B. Population-based analysis of invasive nontypeable pneumococci reveals that most have defective capsule synthesis genes. PLoS One. 2014;9, e97825.

11. Weiser JN, Bae D, Epino H, Gordon SB, Kapoor M, Zenewicz LA, Shchepetov M. Changes in availability of oxygen accentuate differences in capsular polysaccharide expression by phenotypic variants and clinical isolates of Streptococcus pneumoniae. Infect Immun. 2001;69:5430-9.

12. PubMLST database: http://pubmlst.org/. Accessed 3 Jul 2015.

13. Ndlangisa KM, du Plessis M, Wolter N, de Gouveia L, Klugman KP, von Gottberg A. Population snapshot of Streptococcus pneumoniae causing invasive disease in South Africa prior to introduction of pneumococcal conjugate vaccines. PLoS One. 2014;9, e107666.

14. Scott JR, Hinds J, Gould KA, Millar EV, Reid R, Santosham M, O'Brien KL, Hanage WP. Nontypeable pneumococcal isolates among navajo and white mountain apache communities: are these really a cause of invasive disease? J Infect Dis. 2012;206:73-80

15. Salter SJ, Hinds J, Gould KA, Lambertsen L, Hanage WP, Antonio M, Turner P, Hermans PW, Bootsma HJ, O'Brien KL, Bentley SD. Variation at the capsule locus, cps, of mistyped and non-typable Streptococcus pneumoniae isolates. Microbiology. 2012;158:1560-9.

16. Morona JK, Paton JC, Miller DC, Morona R. Tyrosine phosphorylation of CpsD negatively regulates capsular polysaccharide biosynthesis in streptococcus pneumoniae. Mol Microbiol. 2000;35:1431-42.

17. Morona JK, Miller DC, Morona R, Paton JC. The effect that mutations in the conserved capsular polysaccharide biosynthesis genes $\operatorname{cps} A$, cpsB, and $\operatorname{cps} D$ have on virulence of Streptococcus pneumoniae. J Infect Dis. 2004;189:1905-13.

18. Kolkman MA, Wakarchuk W, Nuijten PJ, van der Zeijst BA. Capsular polysaccharide synthesis in Streptococcus pneumoniae serotype 14: molecular analysis of the complete cps locus and identification of genes encoding glycosyltransferases required for the biosynthesis of the tetrasaccharide subunit. Mol Microbiol. 1997;26:197-208.

19. Melchiorre S, Camilli R, Pietrantoni A, Moschioni M, Berti F, Del GM, Superti F, Barocchi MA, Pantosti A. Point mutations in wchA are responsible for the nontypability of two invasive Streptococcus pneumoniae isolates. Microbiology. 2012;158:338-44.

20. Shainheit MG, Valentino MD, Gilmore MS, Camilli A. Mutations in pneumococcal cpsE generated via in vitro serial passaging reveal a potential mechanism of reduced encapsulation utilized by a conjunctival isolate. J Bacteriol. 2015;197:1781-91

21. Madhi SA, Petersen K, Madhi A, Wasas A, Klugman KP. Impact of human immunodeficiency virus type 1 on the disease spectrum of Streptococcus pneumoniae in South African children. Pediatr Infect Dis J. 2000;19:1141-7.

22. Chewapreecha C, Harris SR, Croucher NJ, Turner C, Marttinen P, Cheng L, Pessia A, Aanensen DM, Mather AE, Page AJ, Salter SJ, Harris D, Nosten F, Goldblatt D, Corander J, Parkhill J, Turner P, Bentley SD. Dense genomic sampling identifies highways of pneumococcal recombination. Nat Genet. 2014;46:305-9

23. Huebner RE, Klugman KP, Matai U, Eggers R, Hussey G. Laboratory surveillance for Haemophilus influenzae type B, meningococcal, and pneumococcal disease. Haemophilus Surveillance Working Group. S Afr Med J. 1999;89:924-5.

24. Ruoff KL, Whiley RA, Beighton D. Streptococcus. In: Murray PR, Jorgensen JH, Landry ML, Pfaller MA, editors. Manual of clinical microbiology. Washington DC: ASM Press; 2003. p. 405-21.

25. Wasas AD, Huebner RE, De BM, Klugman KP. Long-term survival of Streptococcus pneumoniae at room temperature on Dorset egg medium. J Clin Microbiol. 1998:36:1139-40.

26. Austrian R. The quellung reaction, a neglected microbiologic technique. Mt Sinai J Med. 1976:43:699-709.

27. Clinical and Laboratory Institute, Wayne: Clinical and laboratory Standards. Perfomance Standards for Antimicrobial Susceptibility Testing. 2010
28. Carvalho MG, Tondella ML, McCaustland K, Weidlich L, McGee L, Mayer LW, Steigerwalt A, Whaley M, Facklam RR, Fields B, Carlone G, Ades EW, Dagan R, Sampson JS. Evaluation and improvement of real-time PCR assays targeting lytA, ply, and psaA genes for detection of pneumococcal DNA. J Clin Microbiol. 2007:45:2460-6.

29. Pimenta FC, Roundtree A, Soysal A, Bakir M, du Plessis M, Wolter N, von Gottberg A, McGee L, Carvalho Mda G, Beall B. Sequential triplex real-time PCR assay for detecting 21 pneumococcal capsular serotypes that account for a high global disease burden. J Clin Microbiol. 2013;51:647-52.

30. Hammerschmidt S, Wolff S, Hocke A, Rosseau S, Muller E, Rohde M. Illustration of pneumococcal polysaccharide capsule during adherence and invasion of epithelial cells. Infect Immun. 2005;73:4653-67.

31. Rissman Al, Mau B, Biehl BS, Darling AE, Glasner JD, Perna NT. Reordering contigs of draft genomes using the Mauve aligner. Bioinformatics. 2009;25: 2071-3.

32. Seemann T. Prokka: rapid prokaryotic genome annotation. Bioinformatics. 2014;30:2068-9.

33. Jolley KA, Maiden MC. BIGSdb: scalable analysis of bacterial genome variation at the population level. BMC Bioinf. 2010;11:595. doi:10.1186/ 1471-2105-11-595:595-11.

34. Page AJ, Cummins CA, Hunt M, Wong VK, Reuter S, Holden MT, Fookes M, Falush D, Keane JA, Parkhill J. Roary: rapid large-scale prokaryote pan genome analysis. Bioinformatics 2015;31:3691-3

35. Stamatakis A. RAxML version 8: a tool for phylogenetic analysis and postanalysis of large phylogenies. Bioinformatics. 2014;30:1312-3.

36. Alikhan NF, Petty NK, Ben Zakour NL, Beatson SA. BLAST Ring Image Generator (BRIG): simple prokaryote genome comparisons. BMC Genomics. 2011;12:402. doi:10.1186/1471-2164-12-402:402-412.

\section{Submit your next manuscript to BioMed Central and we will help you at every step:}

- We accept pre-submission inquiries

- Our selector tool helps you to find the most relevant journal

- We provide round the clock customer support

- Convenient online submission

- Thorough peer review

- Inclusion in PubMed and all major indexing services

- Maximum visibility for your research

Submit your manuscript at www.biomedcentral.com/submit
Biomed Central 